\title{
Cytomorphology of periarticular nodular swellings: One year study
}

\author{
Dharitri Bhat ${ }^{1, *}$, Poonam Malani ${ }^{2}$, Swati Kalantri ${ }^{3}$, Dinkar T. Kumbhalkar ${ }^{4}$, Waman K. Raut ${ }^{5}$ \\ ${ }^{1}$ Associate Professor, ${ }^{2,3}$ Senior Resident, ${ }^{4}$ Professor and Vice Dean, ${ }^{5}$ Professor and Head, Dept. of Pathology, Government \\ Medical College, Nagpur, Maharashtra, India
}

*Corresponding Author:

Email: dharitribhat@gmail.com

\begin{abstract}
Introduction: Periarticular nodular swellings represent one of the common manifestations of a wide variety of non-neoplastic and neoplastic lesions. It poses diagnostic challenge for both the clinicians and pathologists due to overlapping signs and symptoms and nonspecific laboratory findings. FNAC helps in categorizing these lesions as inflammatory, degenerative, benign and malignant and offers an early diagnosis.

Aims and Objectives: To categorise different nodular swellings around synovial joints of upper and lower limbs and to study the Cytomorphology of these lesions.

Materials and Methods: An observational case study of 100 patients with Periarticular nodules of upper and lower extremities referred to the cytology OPD of GMC, Nagpur Maharashtra, over one-year duration.

Results: Commonest lesion found was ganglion cyst in 37\% patients, followed by lipoma in $18 \%$ patients, epidermal cyst in $13 \%$ patients, inflammatory lesions in $11 \%$ patients, giant cell tumor of tendon sheath in $8 \%$ patients, benign mesenchymal tumors in $7 \%$ patients. Other interesting cases included gout in $2 \%$ patients, rheumatoid nodule, ochronosis, xanthoma, nerve abscess, synovial sarcoma, chloroma and metastasis of squamous cell carcinoma in $1 \%$ each.
\end{abstract}

Keywords: Periarticular nodules, FNAC, Inflammatory, Cytomorphology.

\section{Introduction}

Periarticular nodular swellings represent one of the common manifestations of a wide variety of nonneoplastic and neoplastic lesions. In addition to causes of nodular swellings seen in any other location in the body, periarticular tissues are prone to various joint and tendon related pathology such as ganglion cysts, giant cell tumour of tendon sheath and gouty tophus etc. Many of these lesions are associated with overlapping signs and symptoms and nonspecific laboratory findings. Hence, they can prove to be a diagnostic challenge for both clinicians and pathologists. ${ }^{1}$ FNAC is a good primary modality in the study of periarticular nodular swellings. It helps in categorizing the lesion as inflammatory, degenerative, benign, malignant and offers an early diagnosis. Periarticular nodules such as gouty tophus is an important differential diagnosis which can be difficult in cases of atypical presentation in the absence of arthritis and /or hyperuricemia. As fine needle aspiration cytology (FNAC) is becoming a popular clinical practice in diagnosis of soft tissue nodules, it is important for the pathologist to be aware of the microscopic findings and differential diagnosis various periarticular nodular swellings including gouty tophi. ${ }^{1,2}$ FNAC is nowadays a diagnostic test of choice for superficial lesions located over easily accessible portions of the body as well as few pathological lesions in important internal viscera without seeking recourse to more elaborate time-consuming and invasive techniques such as exploration or open biopsy. ${ }^{3}$ FNAC is valuable in identifying the nature of periarticular nodules especially as inflammatory, neoplastic or others like gouty tophi. ${ }^{4}$ It has been increasingly advocated for lesions like ganglion cysts not only as a diagnostic, but also as a therapeutic modality. ${ }^{5}$ As cytology (FNAC) is becoming popular in diagnosis of soft tissue nodules; it is important for the pathologists to be aware of its varied microscopic features. ${ }^{6}$

\section{Aims and Objectives}

Present study was undertaken with following aims and objectives:

1. To study the Cytomorphology of periarticular nodular swellings and

2. To categorise different nodular swellings around synovial joints of upper and lower limbs.

\section{Materials and Methods}

Present study was an observational case study of one-year duration which included patients with periarticular nodules of upper and lower extremities referred to the cytology OPD of GMC, Nagpur Maharashtra, a tertiary health care centre in central India.

Inclusion Criteria: Patients with nodular swellings in the vicinity of synovial joints of upper and lower extremities in males \& females of all age groups were included in the study.

Exclusion Criteria: Patients with diffuse swellings involving joints, bony lesions and swellings over trunk and other parts of body were not included in the study.

Methodology: All patients who were referred to cytology OPD from Surgery, Plastic Surgery or Skin OPD for FNAC were included. After complete clinical details, relevant radiological details, general examination and local examination of the nodules, FNAC was done using 22 or 23 gauze needles with 
aspiration technique. In cystic lesions, contents were aspirated and inspected grossly. Multiple smears were prepared (alcohol fixed and air dried) stained and evaluated by expert cytologists. The cytodiagnosis was offered when diagnostic features were seen, otherwise in lesions such as inflammatory and benign mesenchymal tumors, broad diagnostic category was given. Sub typing was done in excised lesions. Histological correlation was obtained on excised lesions.

\section{Results}

In present study, total 100 patients with periarticular nodular swellings were examined. Amongst them, 58 (58\%) patients were males \& $42(42 \%)$ patients were females. Few patients had multiple nodules (12), in them the largest and most approachable nodule was selected for cytology. Out of the total 100 cases, diagnostic categorization was done in 98 cases and 2 cases were inadequate. Most of the swellings were located on upper limb with maximum swellings around wrist joint, followed by elbow and shoulder. Table 1 shows diagnostic categories which included cystic, inflammatory, benign and malignant neoplastic and inadequate. Cystic lesions were most common i.e. 51. Other categories included benign neoplastic (33), inflammatory (11) and malignant lesions (3).

Most common lesion found was ganglion (37), followed by lipoma (18) and epidermal cyst (13). The inspection of contents of aspirates was very helpful in cystic swellings. In ganglion cyst it is usually mucoid, gelatinous, sticky. In epidermal cyst it is granular whitish pultaceous and often foul smelling. Maximum ganglion cysts were located around wrist joint. Smears showed bluish mucoid material in the background with few scattered round to cuboidal cells showing bland morphology (Fig. 2 A). No specific site predilection was noted for epidermal cyst. Smears showed anucleate squames in clear background (Fig. 2 B). Associated inflammation was also noted in few cases with secondary infection. Histopathological correlation was obtained in $7 / 13$ cases of epidermal cysts.
Smears showing predominantly inflammatory component in the absence of other characteristic diagnostic features were categorized as inflammatory lesions. It included interesting cases such as granulomatous lesion (4), gout (2), fungal granuloma (1), and nerve abscess (1). These granulomatous lesions included 1 case of rheumatoid nodule diagnosed in view of other serological findings and proper clinical setting, and a case of erythema nodosum diagnosed on histopathology. Other two inflammatory lesions, were confirmed as infected epidermal cyst and organizing abscess on histology.

In benign neoplastic category, most common benign tumor was lipoma. In lipoma the aspirate was oily, greasy and smears showed mature adipose tissue fragments with entangled branching capillary vessels (Fig. 2 C). 12/19 lesions were excised, of which 11 were reported as lipoma, however one case turned out to be Xanthoma on further workup. Apart from lipoma, other tumors were giant cell tumor of tendon sheath (8), benign mesenchymal tumors unclassified (5), and one case of benign peripheral nerve sheath tumor which was confirmed on histopathology. Three malignancies were noted in present study, of which one was malignant mesenchymal tumor which turned to be synovial sarcoma on excision and IHC workup. Other malignant lesion was myeloid sarcoma/ chloroma in a known patient of CML on chemotherapy who developed a nodule over shoulder region. Another patient was a known case of squamous cell carcinoma on treatment who presented with a nodule over wrist joint. In two cases which were inadequate for interpretation due to either scanty or non-representative sample, histopathology was done. One case turned out to be ochronosis in view of granulomatous reaction around amorphous brown black material and typical history of black discoloration of urine on exposure to air. Other case was an arteriovenous malformation diagnosed on histology.

Table 2 shows the list of swellings excised and the diagnosis offered on histopathology. In other swellings excision was either not indicated or not done. Thus, histopathological correlation was obtained in 32/34 lesions.

Table 1: List of diagnostic categories in present study

\begin{tabular}{|l|c|c|}
\hline S. No & Diagnostic category & No. of cases \\
\hline 1 & Cystic & 51 \\
\hline & Ganglion (37) & \\
\hline & Epidermal Cyst (13) & \\
\hline & Benign Cystic lesion (1) & 11 \\
\hline 2 & Inflammatory & \\
\hline & Granulomatous (4) & \\
\hline & Gout (2) & \\
\hline & Necrotizing (2) & \\
\hline & Nerve abscess (1) & \\
\hline
\end{tabular}




\begin{tabular}{|l|c|c|}
\hline & Nonspecific (1) & \\
\hline 3 & Benign neoplastic & 33 \\
\hline & Lipoma (19) & \\
\hline & Giant cell tumor tendon sheath (8) & \\
\hline & Benign spindle cell tumor (5), Schwannoma (1) & 3 \\
\hline 4 & Malignant neoplastic & \\
\hline & Primary MMT (1) & 2 \\
\hline & Secondary (2) & \\
\hline 5 & Inadequate & \\
\hline
\end{tabular}

Table 2: Showing list of histopathological diagnosis in excised specimens

\begin{tabular}{|c|c|c|}
\hline Histopathology Done & No of cases & Final Diagnosis \\
\hline Lipoma & $12 / 19$ & 11 lipoma/ 1 tendinous xanthoma \\
\hline Epidermal cyst & $7 / 13$ & All epidermal cyst \\
\hline Giant cell tumor tendon sheath & $5 / 8$ & All GCT \\
\hline $\begin{array}{l}\text { Benign Spindle cell tumor, } \\
\text { schwannoma }\end{array}$ & $3 / 5$ & 2 Benign fibrous Histiocytoma, 1 schwannoma \\
\hline Granulomatous & $2 / 4$ & 1 rheumatoid nodule, 1 erythema nodosum \\
\hline Inflammatory others & $2 / 2$ & 1 infected epidermal cyst, 1 organizing abscess \\
\hline Malignant mesenchymal Tumor & $1 / 1$ & Synovial sarcoma \\
\hline Inadequate & $2 / 2$ & Ochronosis, $1 \mathrm{AV}$ malformation \\
\hline Total & 34 & \\
\hline
\end{tabular}

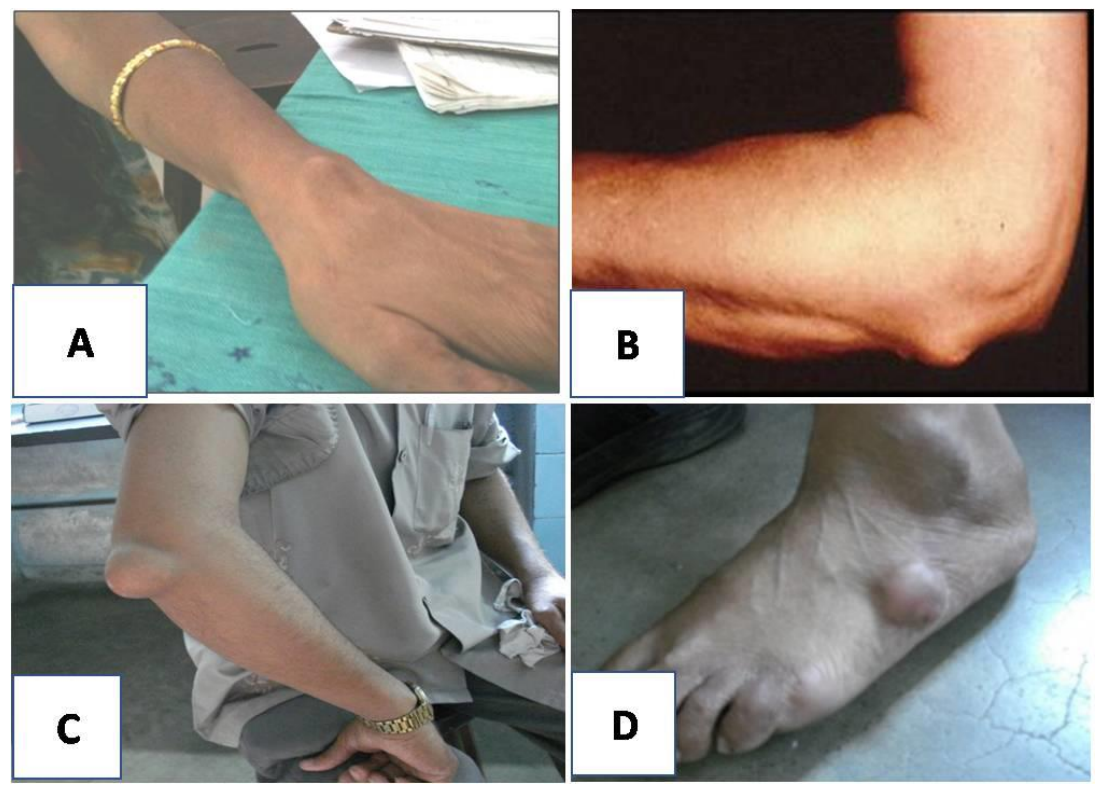

Fig. 1: Photograph showing periarticular nodular swellings 



Fig. 2: Showing cytomorphology of periarticular swellings; (A): Smear showing mucoid gelatinous background and small dispersed cells; (B): Anucleate squames in epidermal cyst; (C): Mature adipose tissue fragments in lipoma; (D): Amorphus material and crystals in Gout

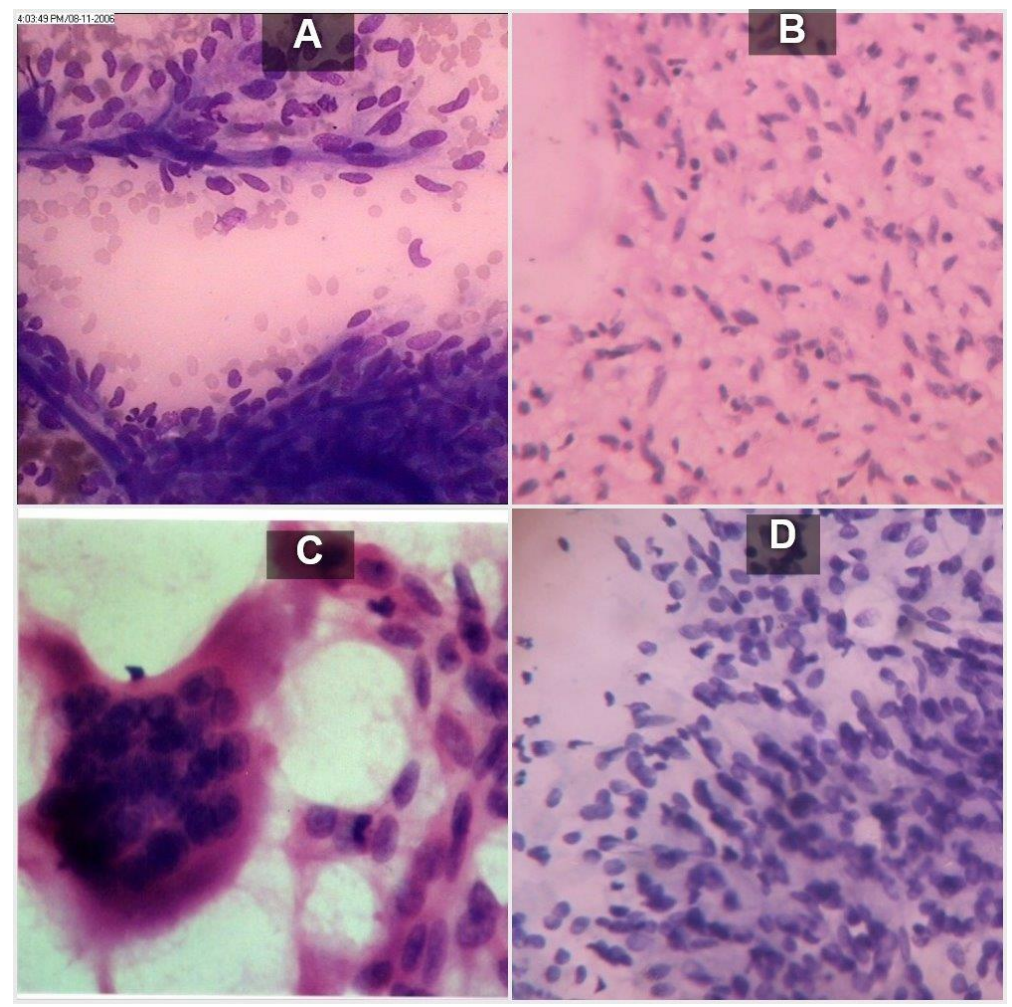

Fig. 3: Showing cytomorphology in neoplastic lesions 


\section{Discussion}

Superficial palpable nodular swellings are very commonly seen in diverse clinical settings. Differential diagnosis of swellings in periarticular location include joint and tendon related pathology in addition to other causes of nodules at other locations. ${ }^{1,2}$ Nonspecific and overlapping sign and symptoms in these swellings often pose a diagnostic challenge to clinicians as well as pathologists. FNAC is a good primary modality in the diagnosis of superficial palpable nodular swellings such as benign soft tissue tumors. ${ }^{7}$ Although there are many case studies on individual diagnostic categories, such type of study on periarticular nodular swellings could not be traced. Present study was conducted in a tertiary care hospital in Central India and it included 100 cases of periarticular nodular swellings around synovial joints of upper and lower limbs with the aim of categorizing the lesions based on their morphology and to study the cytomorphology of these lesions. The FNAC smears were evaluated by expert cytologists. With slight male predominance, most of the swellings were located on upper limb around wrist joint, followed by elbow and shoulder. 98/100 smears were categorized as cystic, inflammatory, benign neoplastic and malignant based on the cytomorphology. Two cases showed inadequate material for assessment.

The contents of the aspirate, colour and other findings such as viscosity, consistency is a helpful clue in the interpretation of these lesions especially cystic lesions like ganglion and epidermal cyst. In the appropriate clinical settings, the diagnosis of ganglion cyst can be made with confidence in cytology. ${ }^{5} \mathrm{We}$ found total 37 ganglion cysts with wrist as the most common location (31/37). Ganglion cyst usually result from mucoid, cystic degeneration of soft tissues adjacent to a joint space. Aspiration of cyst contents has been increasingly advocated as a diagnostic and, in some instances, therapeutic modality. ${ }^{5}$

Next common lesion in cystic category was epidermal cyst. The contents of the aspirate were granular thick, whitish yellow material sometimes foul smelling. No specific site predilection was noted for epidermal cyst. Smears showed anucleate squamous cells in clean background with keratinous material in some. Associated inflammatory component indicates infected epidermoid cyst which is not uncommon. ${ }^{8}$ One case showed clear to thin yellowish aspirate with no specific cytological features for further categorization. This was labelled as benign cystic lesion. Final diagnosis was not obtained in this case as it was not excised.

The category of inflammatory lesions included 11 cases in which smears showed variable number of inflammatory cells in the absence of other specific findings of neoplastic or cystic lesions. Many interesting findings were noted in this category. In presence of epitheloid cells and giant cells, they were labelled as granulomatous inflammation. Two cases also showed significant necrosis in the background. Special stains could not demonstrate Acid Fast Bacilli so these were labelled as necrotizing granulomatous inflammation. In a case of nodule over finger, smears showed branching hyphal forms which were highlighted on PAS stain. This was reported as fungal granuloma. Pheohyphomycosis was confirmed on fungal culture. Demonstration of fungal forms on smears makes FNAC very sensitivity and specificity of the test. ${ }^{9}$

FNAC smears from a nodule near elbow showed necrotic inflammatory background. Since the patient was a known case of leprosy, special stain was performed which demonstrated plenty of lepra bacilli. Hence a diagnosis of nerve abscess was given in this clinical setting. In case of clinical suspicion of pure neuritic leprosy, careful search for nerve elements in addition to necrosis and inflammation is often helpful in arriving at the diagnosis. There are a significant number of studies and case reports emphasizing the clinical utility of FNAC in neuritic leprosy. ${ }^{10}$ One granulomatous inflammation was diagnosed as erythema nodosum on histopathology. While in one case, patient was a known case of rheumatoid arthritis with RA factor positivity. Histopathology confirmed rheumatoid nodule. Subtyping in such cases on FNAC may be limited due to nonspecific and overlapping morphology.

In 2 cases, smears from ankle and toe swelling showed the presence of needle shaped crystals and many giant cells in inflammatory background, which raised the suspicion of gouty tophus (Fig. 2 D). Uric acid levels were also found raised after suspicion of gout on FNAC. So, the diagnosis of Gout was offered. In case of gout, the advantage of FNAC over excision biopsy is that urate crystals are often lost during histological processing because they dissolve in formalin, but they are preserved in FNAC smears that are alcohol fixed. ${ }^{11}$ The crystal demonstration in FNAC smears is superior to histopathology sections where crystals are more commonly lost during processing. ${ }^{12}$ One potential pitfall in aspiration cytology is distinguishing the contents of a gouty tophus from a deposit of pseudo gout. Although it is less common than gout, chondrocalcinosis, or pseudo gout, can mimic gout both clinically and histologically. Careful examination of the crystals will reveal distinct differences between the two processes: In gout crystals are needle shaped with pointed ends whereas calcium pyrophosphate crystals have blunted or squared ends. Also, gouty crystals are strongly (negatively) birefringent where pseudo gout crystals display weak (positive) birefringence on polarization. ${ }^{13}$

Benign soft tissue tumors quiet commonly present as subcutaneous palpable nodules. The incidence of benign soft tissue tumors is about ten times that of malignant ones. Benign tumors are usually superficial and well defined or encapsulated masses showing slow 
growth. In our study, 19 lipomas were diagnosed on cytology. This is the most commonly encountered swelling that is subjected to FNAC. Clinical findings of the swelling are essential in its differentiation from subcutaneous fibro-adipose tissue, accurate needle placement within the mass being the most important criterion. Lipomas are usually solitary but may be multiple, and the swelling slips under the examining hand. The aspirate is usually oily, greasy and show adipose tissue which is indistinguishable from normal fibro adipose tissue. Delicate vessels without much fibrous tissue are the rule. The fat cells are univacuolated with nuclei pushed to the periphery. The presence of cells with atypical nuclei indicates the necessity for histological examination for proper evaluation. Amongst total 19 cases, 12 were excised and all were confirmed on histology except in one case from nodule over elbow, which showed vacuolated xanthoma cells, histiocytes, giant cells and perivascular inflammation. This patient had multiple nodules mainly in periarticular location and hyperlipidemia was also detected later on after suspicion. Scanty aspirate showing vacuolated cells was probably misinterpreted as Lipoma on cytology. Keeping in mind the FNAC features of various conditions is definitely helpful in such cases to avoid misinterpretation.

Next common entity in benign neoplastic lesions was giant cell tumor of tendon sheath (8). They were seen as small firm nodules commonly around fingers and wrist. It is still uncertain whether it represents a true neoplasm or a reactive proliferation in response to trauma. However, its typical clinical and cytological features permit definitive diagnosis on cytology (Fig. 3 C). Classical location and radiological demonstration of the lack of bone involvement is useful in diagnosis. Aspirates show multinucleated giant cells of osteoclastic cell type and two kinds of stromal cells: spindle-shaped cells and polygonal cells with pale cytoplasm. ${ }^{14,15}$

Benign mesenchymal tumors included 5 cases. This included the smears with variable cellularity showing spindle cells with pale cytoplasm and bland looking chromatin with or without interspersed collagen (Fig. 3B). Aspirates may not show the typical findings for subtyping. It is enough to make a distinction from malignancy on FNAC rather than give a precise histological diagnosis. In any aspirate from a spindle cell lesion, the main criteria to be assessed are cellularity, nuclear pleomorphism, mitosis, and necrosis. Sub typing of 2 cases was done on histopathology. Aspirates not showing definite necrosis or any mitosis or clearly pleomorphic nuclei or very high cellularity should be carefully evaluated to distinguish a low-grade sarcoma from a benign spindle cell lesion. Even the slightest increase of cellularity or nuclear chromatin abnormality in the absence of specific features for one of the above tumors, should mandate a histological examination. The purpose of
FNAC in benign soft tissue lesions is to be an initial screening test to differentiate from infective lesions and it should be used to triage patients requiring early biopsy. The specific diagnosis is almost always made on histopathology and FNAC should not be used as a replacement for an excision biopsy. ${ }^{16}$ In one case patient had pain during FNAC. Smears showed, cellular fragments with wavy, buckled nuclei and bland morphology (Fig. 3 A). This was diagnosed as benign nerve sheath tumor and was confirmed as schwannoma on excision. Certain studies mention the presence of features corresponding to Antony A and B areas like variable cellularity and nuclear palisading along with myxoid background. ${ }^{17}$

We encountered with 3 cases of malignancies in our study, of which one was reported as malignant mesenchymal tumor which showed abundant cellularity of spindle to plump pleomorphic cells in blood mixed necrotic background. In view of nuclear hyperchromasia, coarse chromatin and occasional mitosis, the diagnosis of malignant mesenchymal tumor was offered (Fig. 3D). On histology, after complete IHC work up, it was confirmed as synovial sarcoma. Other two cases were known patients of malignancy (squamous cell carcinoma lung and Chronic Myeloid leukemia) on treatment. FNAC confirmed the metastatic deposits in Squamous cell carcinoma. In case of chloroma, myeloperoxidase stain was very helpful in confirming the myeloid series cells.

In two cases reported as inadequate on cytology, one patient was not willing for repeat aspiration. On excision, granulomatous reaction was seen around amorphous brown black material. Typical history of black discoloration of urine on exposure to air was obtained so it was diagnosed as ochronosis after complete clinical workup. In case of arteiovenous malformation, FNAC was inconclusive even on repeat FNAC. The diagnosis was done in correlation with radiological features after excision.

\section{Conclusion}

In this observational case study of cytomorphology of periarticular nodular swellings, 98/100 cases were categorized on FNAC based on the cytological features. Various categories included cystic (ganglion, epidermal), inflammatory lesions including granulomatous lesions, gout, fungal granuloma and nerve abscess), benign soft tissue tumors (lipomas, giant cell tumor of tendon sheath, spindle cell tumors, schwannoma) and malignancies including one primary MMT and two metastases in known patients with malignancy. Histological correlation was obtained in excised specimens (32/34). Since differential diagnosis of nodular swellings in periarticular location include spectrum of all lesions such as degenerative, inflammatory and neoplastic, awareness about the cytomorphology of these lesions is essential in proper categorization of these nodular swellings. 


\section{References}

1. Dodd LG, Major NM: Fine Needle aspiration cytology of articular and periarticular lesions. Cancer, 2002;96:157165.

2. De Haas WH et al, Synovial nature of pathologic periarticular structures including subcutaneous nodules, descent from embryonic arthrogenic fibroblast: a hypothesis. Z rheumtolo. 1979;38(9-10):318-293.

3. Hoogar MB, Wani AS, Bhemat DN, Borkar DB, Tambekar M, Dhar R, Fine needle aspiration cytology (FNAC) of periarticular odules- an astonishingly effective tool in the diagnosis of tophaceous gout. Indian and Applied Medical Research. 2016;5(3):532-536.

4. Rathod GB, Annie Jain, Role of FNAC in diagnosis of gouty tophi, A case report. IAIM. 2015;2(7):137-140.

5. Dodd LG, Layfield LJ, Fine Needle Aspiration Cytology of ganglion cysts. Diagn Cytopathol.1996;2015;2(7):137140

6. Hanmante RD, Mulay PS, Kadam PN, Diagnostic utility of FNAC in gouty tophi with review of literature. IAIM, 2015;2(2):123-128.

7. Iyer VK, Cytology og soft tissue tumors: Benign soft tissue tumors including reactive, nonneoplastic lesions. Journal of Cytology. 2008;25(3):81-86.

8. Handa u, Chhabra S, Mohan H, epidermal Inclusion cyst: cytomorphological features and differential diagnosis. Diagn Cytopathol. 2008;36(12):861-3.

9. Das DK, Grover RK, Chachra KL, Bhatt NC, Misra B. Fine needle Aspiration Cytology Diagnosis of a fungal Lesion of the Verticillium Species; Acta Cytologica. 1997;41:577-82.

10. Kiran CM, Menon R, Fine needle aspiration cytology as an aid to diagnosis, categorization and treatment when pure neuritic leprosy presents as nerve abscess. J Cytol. 2013;30(4): 237-240.

11. Walke V, Ramraje S, Jadhao V, Cytodiagnosis of gouty tophus. Cytojournal. 2013:10:11.

12. Agrawal K: Pahuja S; Agrawal C; Harbhajanka A; FNAC of gouty tophi with review of literature. Journal of cytology. 2007;24(3):142-145.

13. Allen EA, Ali SZ, Erozan YS, Tumoral calcium pyrophosphate dihydrate deposition disease: Cytopathologic findings on fine needle aspiration. Diagn Cytopathol. 1996;15,349-351.

14. Iyer VK, Kapila K, Verma K. Fine needle aspiration cytology of giant cell tumor of tendon sheath. Diagn Cytopathol. 2003;29:105-1

15. Paul E. Wakely, Jr, MD William J. Frable, MD; Fineneedle Aspiration Biopsy Cytology of Giant-cell tumor of Tendon Sheath; American Journal of Clinical Pathology. 1994;102(1):87-90.

16. Costa MJ, Campman SC, Davis RL, Howell LP. Fineneedle aspiration cytology of sarcoma: Retrospective review of diagnostic utility and specificity. Diagn Cytopathol. 1996;15:23-32.

17. Kim SY, Nam HJ, Kim JH, Cho YR, Kang YN, Lee SS Fine Needle Aspiration Cytologic Features of Schwannomas. The Korean Journal of Cytopathology. 1999;10(1):55-60.

How to cite this article: Bhat $\mathrm{D}$, Malani $\mathrm{P}$, Kalantri S, Kumbhalkar DT, Raut WK. Cytomorphology of periarticular nodular swellings: One year study. J Diagn Pathol Oncol. 2018;4(3):180-186. 